Supporting Information for:

\title{
Recrystallized Perovskite Thin Film via Intense Pulse Light Sintering for Vertical Gradient Bandgap Perovskite Solar Cells
}

\author{
Shiuan-Ying Peng, ${ }^{*} \quad$ Tzu-Sen Su, ${ }^{\S}$ Cheng-An Chen,, Kai-Wen Chuang, ${ }^{\dagger}$ Tzu-Chien Wei, ${ }^{*}$, \\ Ying-Chih Liao ${ }^{*}$,
}

Department of Chemical Engineering, National Taiwan University, Taipei 10617, Taiwan

$\S$ Department of Chemical Engineering, National Tsing Hua University, Hsinchu 300, Taiwan

* Corresponding author, E-mail: $\underline{\text { liaoy@ntu.edu.tw }}$

Phone number: $+886-2-3366-9688$

\section{Contents:}

I. Mutual dissolution

II. $\mathrm{MAPbBr}_{3}$ quantum dots with and without UV excitation / IPL light source spectrum

III. Energy density measurement

IV. Penetration depth of IPL

V. Depth analysis of the stacked perovskite film

VI. Photoluminescence spectra of stacked perovskite film with different sintering conditions

VII. Photoluminescence spectrum of gradient perovskite

VIII. Photoluminescence spectra of stacked perovskite films with different power and excitation time

IX. Band gap energy

X. The image of degraded perovskite film with and without IPL sintering 


\section{Mutual dissolution}

1. Drop casting or spin coating

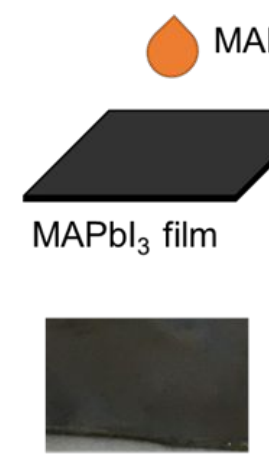

Pristine $\mathrm{MAPbl}_{3}$ film

$\mathrm{APbBr}_{3}$ droplet
2. Droplet dissolved the film

3. Recrystallization
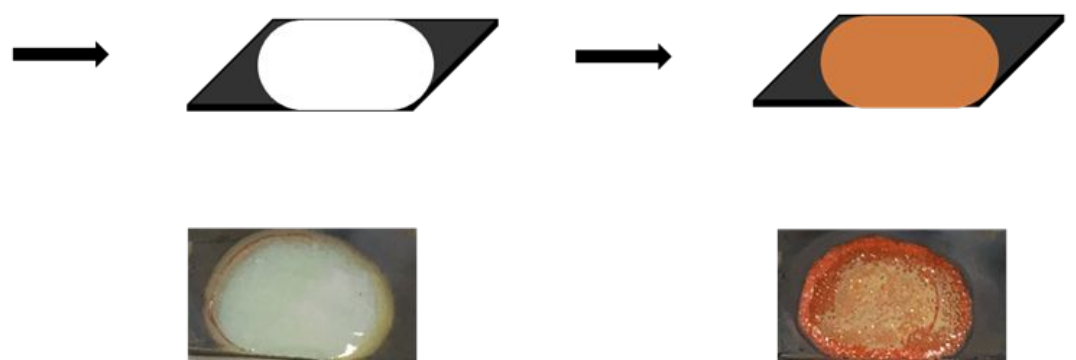

Droplet dissolved the $\mathrm{MAPbl}_{3}$ film

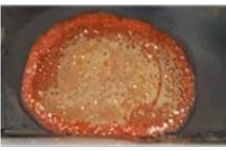

$\mathrm{MAPbl}_{3-\mathrm{x}} \mathrm{Br}_{\mathrm{x}}$ film

Figure S1. Schematic of the wet process by drop casting $1 \mathrm{M} \mathrm{MAPbBr}$ and $1 \mathrm{M} \mathrm{MAPbI}_{3}$. Below the schematic is the practical crystallization process. 
II. MAPbBr 3 quantum dots with and without UV excitation/ IPL light source spectrum

(a)

(c)

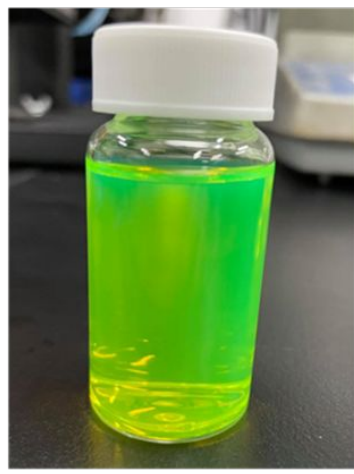

(b)
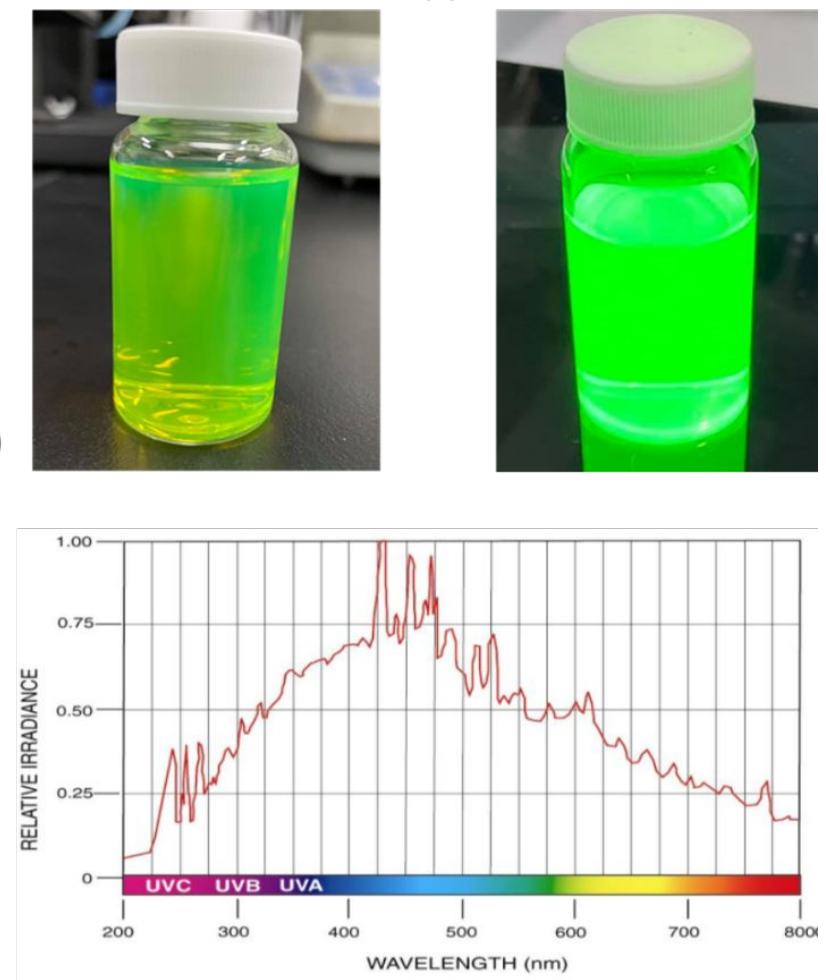

Figure S2. Photographs of (a) the $\mathrm{MAPbBr}_{3}$ QD solution and (b) the $\mathrm{MAPbBr}_{3}$ QD solution under UV excitation. (c) Spectrum of the IPL light source. 
III. Energy density measurement

Table S1. The energy densities of Xenon X-1000 (IPL machine)

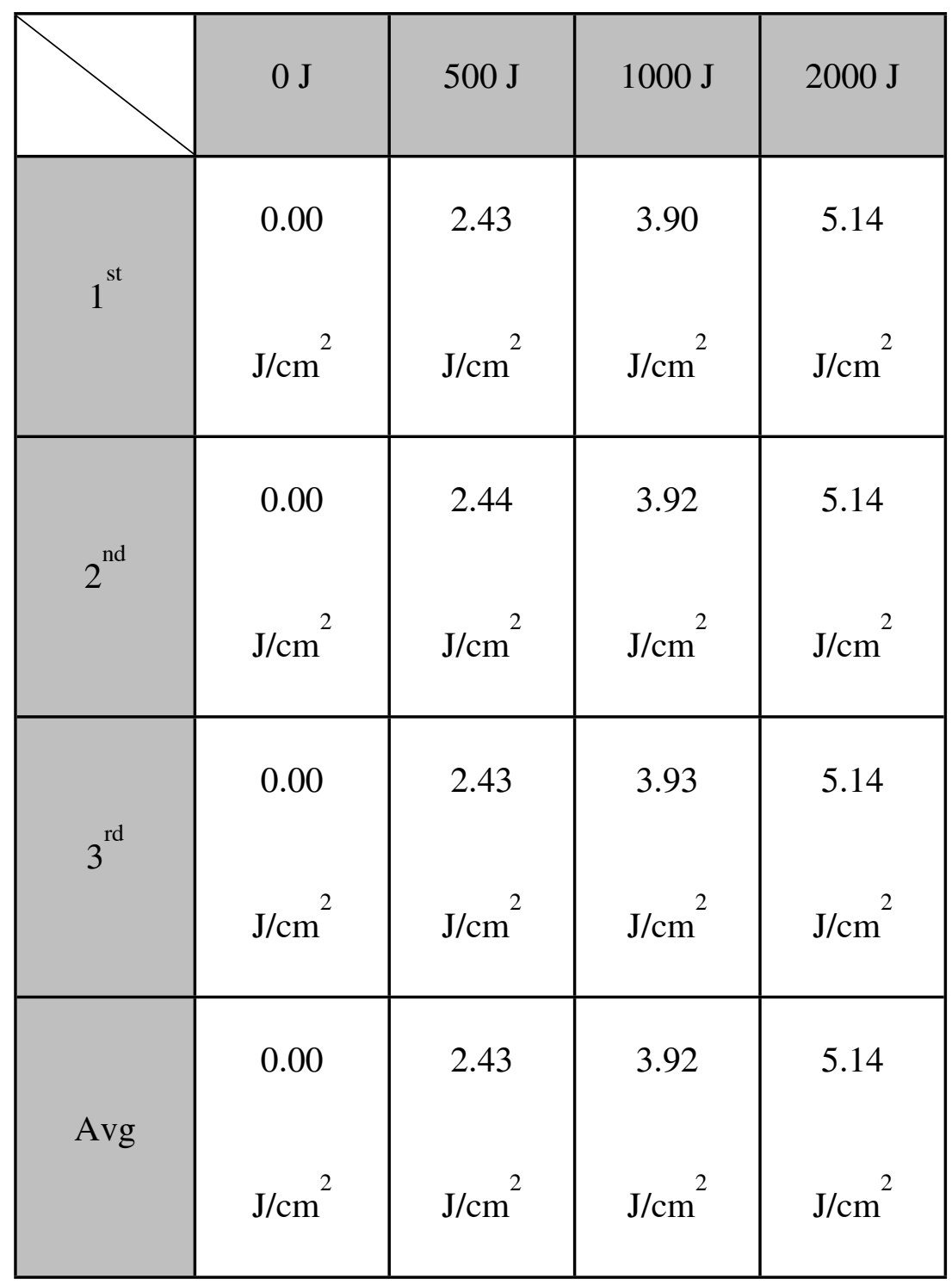

Detector: Ophir NOVA II @Working Distance= $2 \mathrm{~cm}$ and $3000 \mathrm{~V}$ 
IV. Penetration depth of IPL

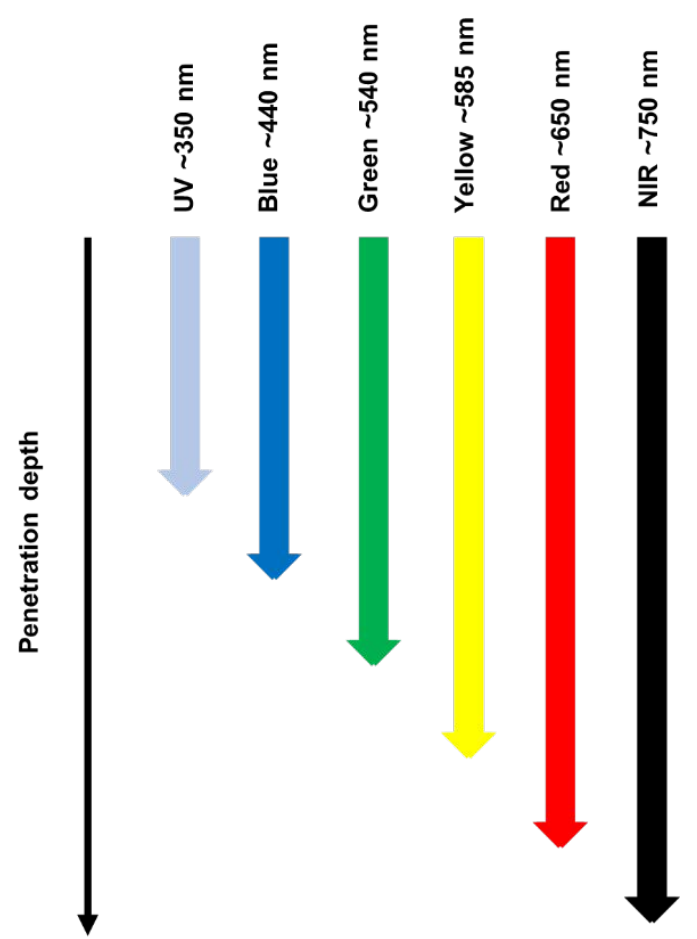

Figure S3. The penetration depth of IPL. 
V. Depth analysis of the stacked perovskite film

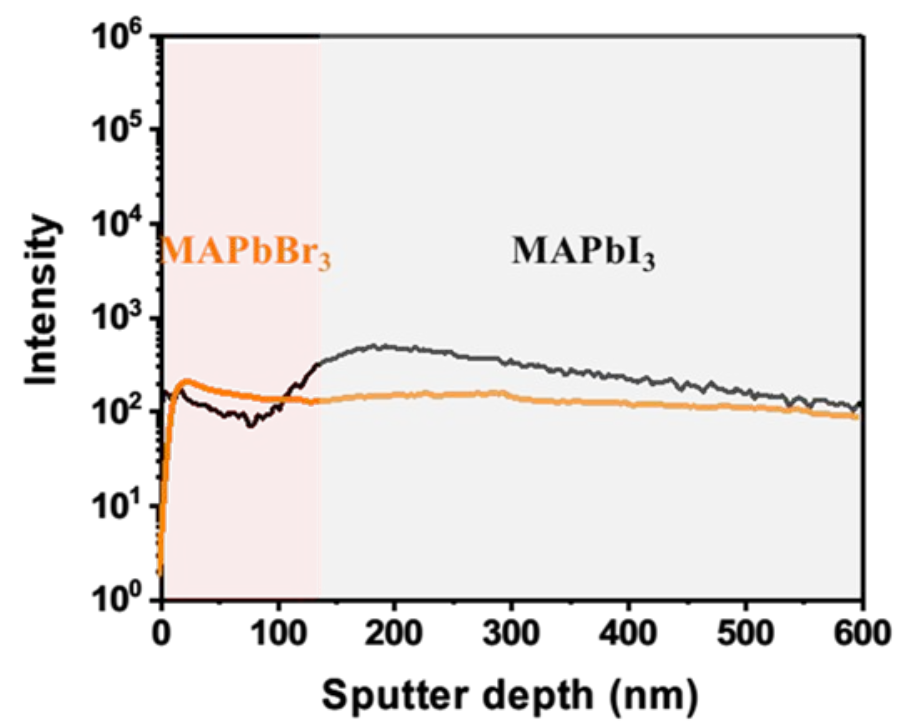

Figure S4. The depth analysis of $\mathrm{MAPbBr}_{3}$ and $\mathrm{MAPbI}_{3}$. 
VI. Photoluminescence spectra of stacked perovskite film with different sintering conditions

(a)

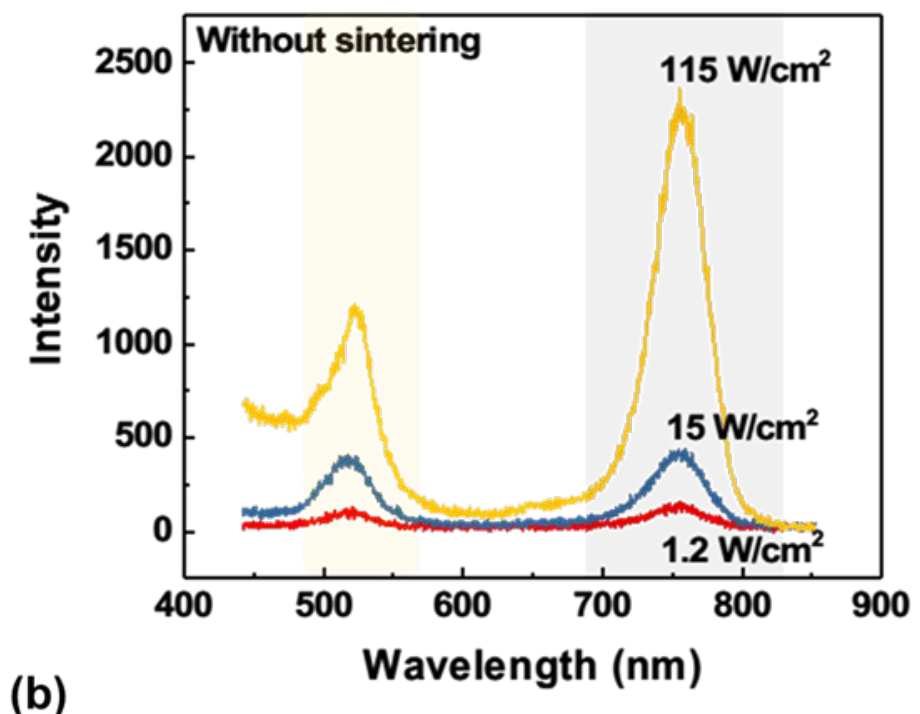

(b)

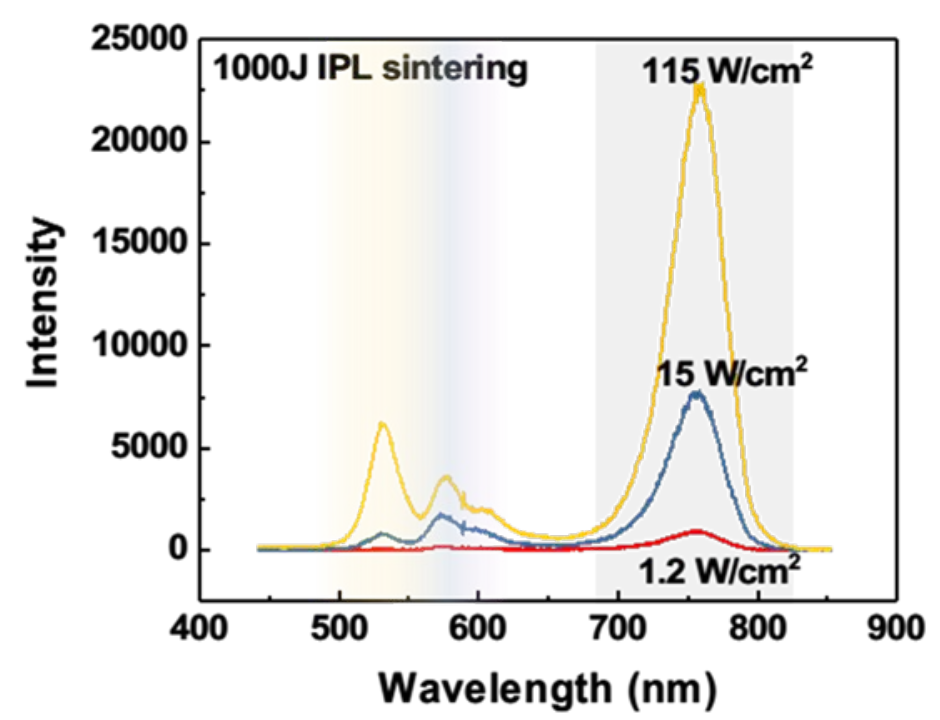

Figure S5. PL spectra of stacked perovskite film as a function of laser power $(405 \mathrm{~nm})$ over $20 \mathrm{~s}$ of continuous-wave laser illumination at room temperature. 
VII. Photoluminescence spectrum of gradient perovskite

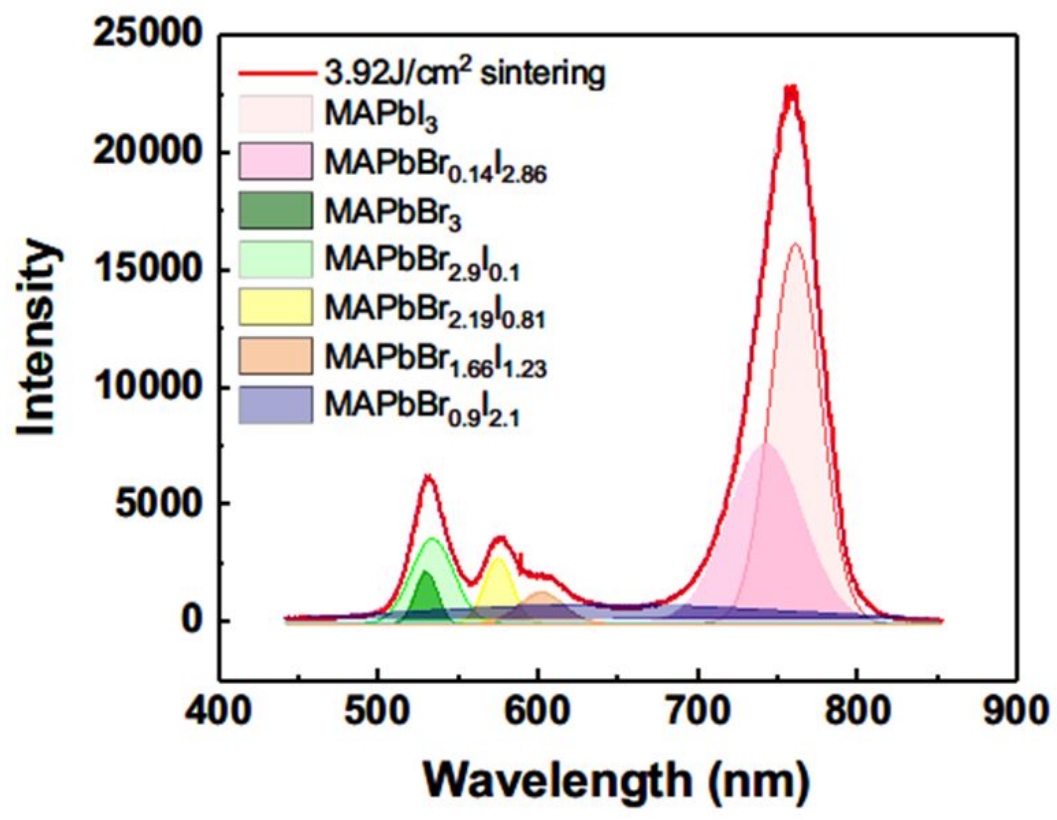

Figure S6. The photoluminescence spectrum of gradient perovskite can be clearly distinguished from various mix halide perovskite emission peaks by using a $405 \mathrm{~nm}$ laser at $115 \mathrm{~mW} / \mathrm{cm}^{2}$.

The composition of mixed-halides perovskite films was estimated by the following relation, $\operatorname{Eg}(\mathrm{x})=1.57+0.39 \mathrm{x}+0.33 \mathrm{x}$. where $\mathrm{E}_{\mathrm{g}}$ is the band gap of the perovskite in $\mathrm{eV}$ and $\mathrm{x}$ is the amount of Br relative to the amount of $\mathrm{I}$. 
VIII. Photoluminescence spectra of stacked perovskite films with different power and excitation time

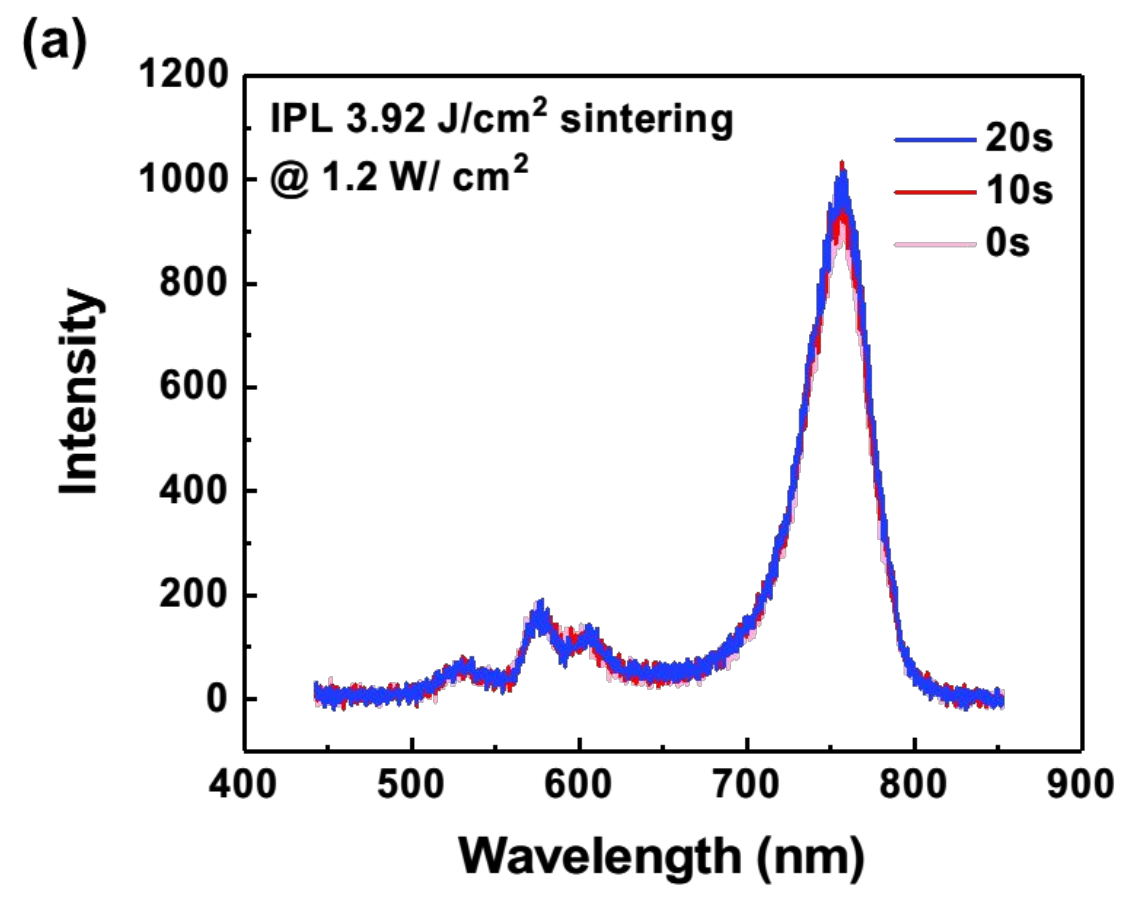

(b)

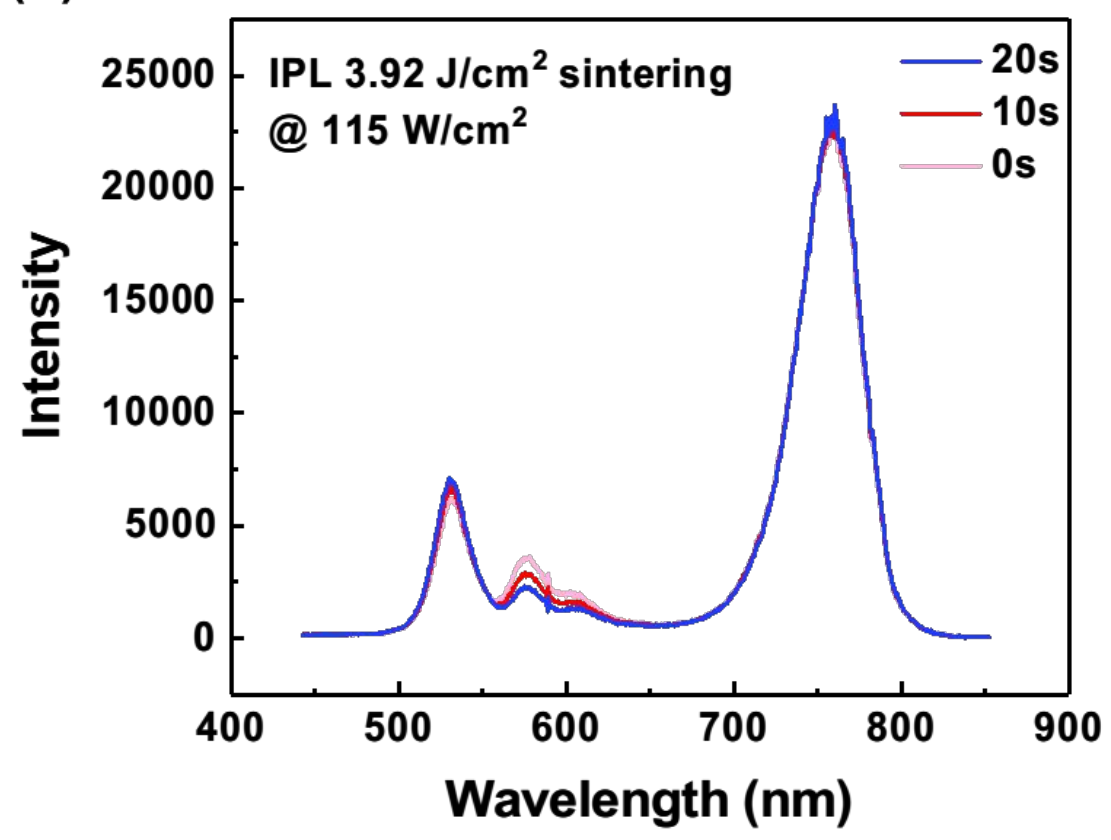

Figure S7. Evolution of the PL spectra of stacked perovskite films with $3.92 \mathrm{~J} / \mathrm{cm}^{2} \mathrm{IPL}$ sintering under excitation intensities of (a) $1.2 \mathrm{~W} / \mathrm{cm}^{2}$ and (b) $115 \mathrm{~W} / \mathrm{cm}^{2}$ during the $0 \mathrm{~s}$ to $20 \mathrm{~s}$ of illumination. 
IX. Band gap energy

(a) Before IPL sintering

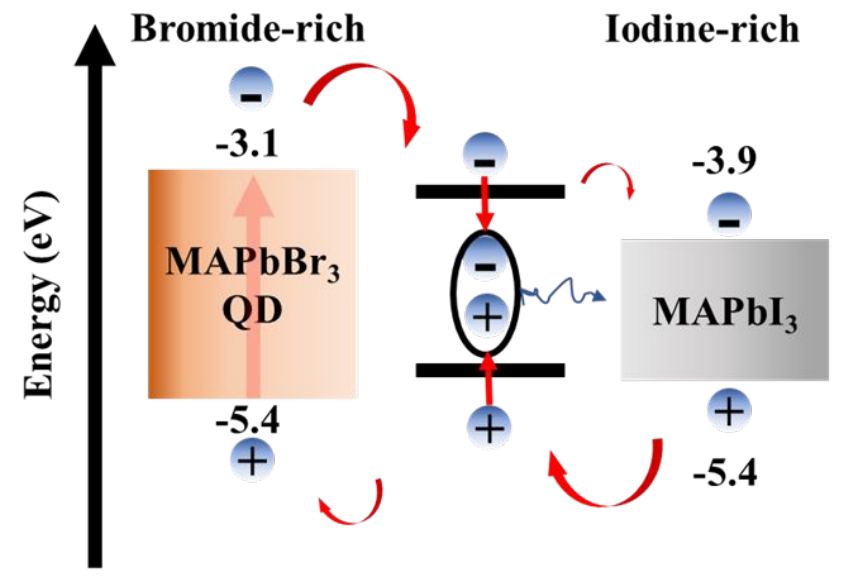

(b) After IPL sintering

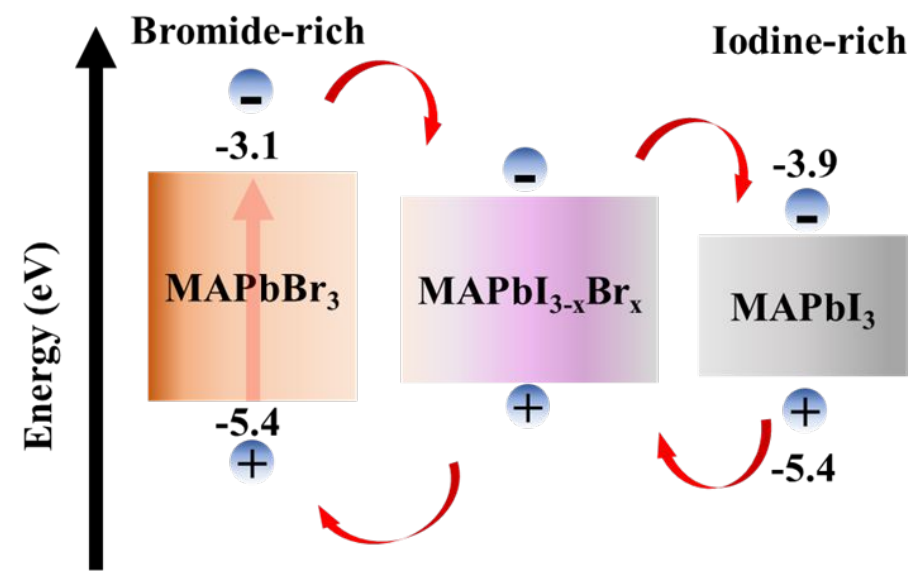

Figure S8. The energy band alignment of (a) the $\mathrm{MAPbBr}_{3} \mathrm{QD} / \mathrm{MAPbI}_{3}$ and (b) the $\mathrm{MAPbBr}_{3}$ $\mathrm{QD} / \mathrm{MAPbI}_{3-\mathrm{x}} / \mathrm{MAPbI}_{3}$ heterostructure material. 
X. The image of degraded perovskite film with and without IPL sintering

(a)

(b)
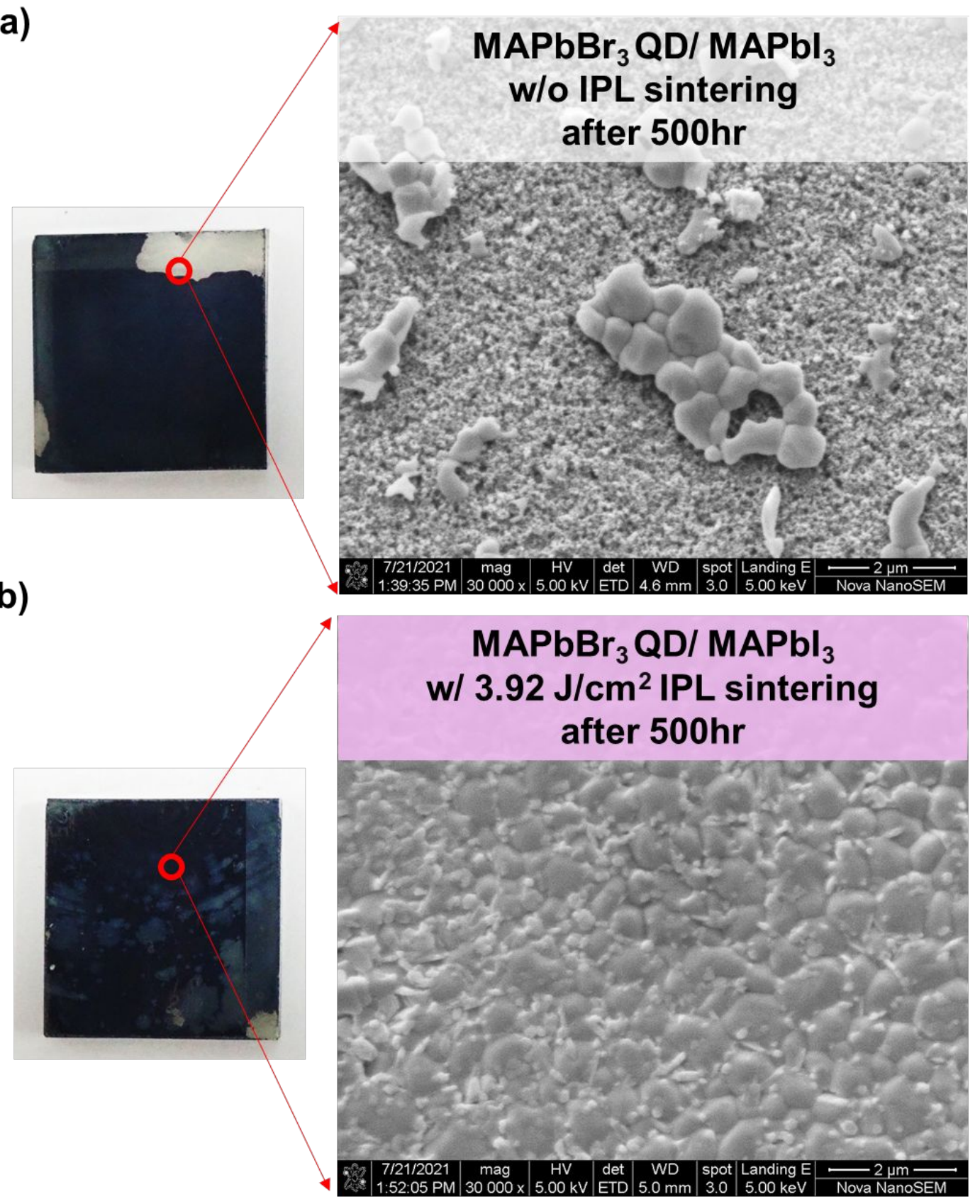

Figure S9. The degradation image of stacked perovskite film (a) without IPL sintering and (b) with $3.92 \mathrm{~J} / \mathrm{cm}^{2}$ under $\mathrm{RH}=70 \%$ and $\mathrm{T}=25^{\circ} \mathrm{C}$ after $500 \mathrm{hr}$. 


\section{References}

1 M. Ibrahim, J. Claudel, D. Kourtiche, M. Nadi, Journal of Electrical Bioimpedance. 2013;4,1322.

2 W. Wechsatol, S. Lorente, A. Bejan, International Journal of Heat and Mass Transfer. $2002 ; 45,4911-24$.

3 A. H. Reis, A. F. Miguel, M. Aydin, Medical Physics. 2004;31,1135-40.

4 A. Miguel, Journal of Applied Fluid Mechanics. 2019;12,1223-9.

5 X.-Q. Wang, A. S. Mujumdar, C. Yap, Journal of Applied Physics. 2007;102,073530.

6 X.-Q. Wang, P. Xu, A. S. Mujumdar, C. Yap, International Journal of Thermal Sciences. $2010 ; 49,272-80$. 